\section{On the brain}

\section{Guy Goodwin}

Journal of Psychopharmacology. Managing editor R. Maggs. Oxford University Press. 4/yr. UK £50, EEC £54, North America $\$ 108$, elsewhere $£ 60$.

Neuropsychopharmacology. Editor-inchief J. Christian Gillin. Elsevier. 6/yr. US\$148, elsewhere $\$ 172$ (institutional); US $\$ 96$, elsewhere $\$ 120$ (personal); US $\$ 34$ elsewhere $\$ 58$ (student).

Pharmacology means far more to psychiatry than just treatment with drugs, in that pharmacological methods provide a way of analysing brain mechanisms and so of identifying abnormalities of transmitter function in psychiatric illness. Other disciplines within the neurosciences, and now also molecular genetics, are

competing to illuminate the nature of mental illness; but pharmacology, by linking therapeutics on the one hand and receptor function on the other, is still the most important of the sciences basic to psychiatry.

\section{Special needs}

\section{J. Marshall}

Molecular Carcinogenesis. Editors-inchief Thomas J. Slaga and Stuart H. Yuspa. Alan R. Liss. 6/yr. US \$190, elsewhere $\$ 241$ (institutional); US $\$ 90$, elsewhere $\$ 120$ (personal).

Although the best papers on oncogenes and molecular aspects of carcinogenesis can still be assured of publication in the high-profile general journals, the huge increase in work in this area and the dictates of editorial fashion have created a need for specialist journals. To my knowledge, Oncogene and Oncogene Research were the first of this new generation, and these journals are now well established.

What then of the relative newcomer Molecular Carcinogenesis, which was started in 1988? Its editors-in-chief propose that it should be a forum for papers describing molecularly orientated research which has direct biological implications. Such an aim is highly laudable, and achieving it looks likely given the distinguished editorial board which draws in experts from many different aspects of cancer research.

On the evidence of the material that has
It is not surprising to find new journals devoted to a fusion between pharmacology, psychology and psychiatry. Furthermore, there is little doubt that there is good work to publish. The problem, as for all multi-disciplinary journals, is to maintain focus and avoid being a repository for research that is not quite good enough to be accepted by the mainstream journals in pharmacology and psychiatry. Journal of Psychopharmacology and Neuropsychopharmacology both have the advantage of originating in societies: the British Association for Psychopharmacology for the first and the American College of Neuropsychopharmacology for the second. Both are now in their second volumes; how are they doing?

Journal of Psychopharmacology aims to publish editorials, reviews, original papers, book reviews and abstracts of meetings. Its contents page does not make a clear distinction between reviews and original papers which may be because it has clearly had difficulty in attracting good-quality research reports. Indeed, the appearance of numbers 3 and 4 for 1988 in a single issue containing only two full papers with original material, looks dangerously like bankruptcy. A particular problem for the journal as a BritishEuropean publication is that two other direct competitors appeared at about the same time (Human Psychopharmacology,

been published to date, the journal obviously has some overlap with Oncogene and Oncogene Research. Topics such as oncogene activation in human tumours and experimental systems, and changes in gene expression in tumours, provide common ground. There are, however, distinct differences in scope. Reflecting the background of many of the editorial board members, there is a strong representation of studies on chemical carcinogenesis. This is very appropriate, given the significance of chemical carcinogenesis in the induction of human malignancy and the importance of chemical carcinogenesis models in animals in providing reproducible systems in which oncogene activation can be studied. In contrast, there is little coverage of topics such as the biochemical functions of oncoproteins.

Molecular Carcinogenesis therefore mainly provides a perspective on how genetic changes may arise in tumour cells rather than on how those changed genes alter all behaviour. Such a perspective is clearly valuable, and the journal should provide an excellent place for the publication of work in this area.

C. J. Marshall is in the Section of Cell and Molecular Biology, Institute of Cancer Research, Chester Beatty Laboratories, Fulham Road, London SW3 6JB, UK published by Wiley, and International Journal of Psychopharmacology, from Clinical Neuroscience Publishers). Success may ultimately depend upon the vitality of its parent association.

Neuropsychopharmacology is a direct American equivalent to Journal of Psychopharmacology, not only in originating in a learned society but in having similar aims and similar composition. It has maintained a higher rate of publication of original papers, although some of these on closer inspection appear to be re-mixes of similar data published elsewhere. Nevertheless, the journal is attracting reasonable basic-science articles and good clinically orientated papers. It clearly benefits from the swing to biologically orientated research in American psychiatry.

Guy Goodwin is in the MRC Brain Metabolism Unit, Royal Edinburgh Hospital, Edinburgh EH10 5HF, UK.

\section{Protein produce}

\section{W.R. Arathoon}

Cytotechnology: International Journal of Cell Culture and Biotechnology. Managing editors J.B. Griffiths, D.W. Barnes and H. Murakawi. Kluwer. 6/yr. Dfl.552, $\$ 258$ (institutional); DfI.270, $\$ 128$ (individual).

ANIMAL-cell culture and related cell biology technologies are taking an increasingly high profile. There is growing demand for the complex proteins from animal cells, be they antibodies, hormones, growth factors, enzymes or vaccines, which are now often made using recombinant DNA techniques. Cytotechnology addresses the needs of this new field, dealing with both

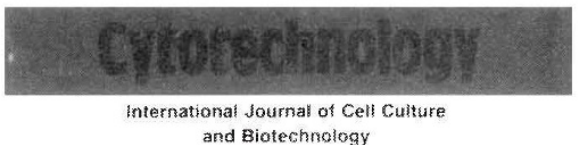

the pure and the applied aspects. No other journal has been directed specifically to this end.

All aspects of normal and transformed cells and hybridomas are covered. Articles range from descriptions of the use of in situ hybridization techniques (of cells and chromosomes) to accounts of new equipment for exploiting cells and characterizing and purifying their products. Probably not to be found in Cytotechnology are reports of the most recent advances in molecular cell biology, which vie for space in the fast-moving, prominent journals. Rather, here you can discover new ways of generating or activating cell lines, new growth-factor activities, more efficient culture systems and formulations for better media.

Cytotechnology contains no glossy 